\title{
Lack of Sexual Identity Disclosure in China May Distort Efforts to Combat STI Epidemics
}

\section{CURRENT STATUS: UNDER REVIEW}

BMC Public Health $\mathbf{B} M C$ Series

Navin Kumar

Yale University

navin.kumar@yale.eduCorresponding Author

ORCiD: https://orcid.org/0000-0003-4502-069X

Laura Forastiere

Yale University

Tiange Zhang

Washington University in Saint Louis School of Medicine

Fan Yang

University of North Carolina at Chapel Hill

Katherine $\mathrm{T} \mathrm{Li}$

Massachusetts General Hospital

Weiming Tang

University of North Carolina at Chapel Hill

Joseph D Tucker

University of North Carolina at Chapel Hill

Nicholas A Christakis

Yale University

Marcus Alexander

Yale University

\section{DOI:}

10.21203/rs.3.rs-15556/v1

\section{SUBJECT AREAS}

Health Economics \& Outcomes Research Health Policy

\section{KEYWORDS}


MSM; Sexual Health; Sexual Identity Disclosure; China 
Abstract

Background. To evaluate whether Chinese men who have sex with men (MSM) select an STI test (rectal vs urethral) appropriate for their sexual behavior (insertive and/or receptive role in anal sex). Methods. We studied uptake of gonorrhea and chlamydia testing among Chinese MSM $(\mathrm{N}=431)$ in a multi-site RCT (December 2018 to January 2019). We collected socio-demographics, relevant medical and sexual history, and disclosure of sexual identity (outness). We estimated the decision to test and test choice, and the extent to which disclosure plays a role in decision-making.

Results. Among 431 MSM, mean age was 28 years $(S D=7.10)$ and $65 \%$ were out to someone. MSM who indicated the versatile role and were out to someone had a $26.8 \%(95 \% \mathrm{Cl}=6.1,47.5)$ increased likelihood for selecting the rectal test vs the urethral test, compared to those versatile and not out. Versatile MSM out to their health provider outside of the study context had a $29.4 \%(95 \% \mathrm{Cl}=6.3$, 52.6) greater likelihood for selecting the rectal STI test vs the urethral test, compared to versatile MSM not out to their health provider.

Conclusions. Anal sex role and identity disclosure may affect gonorrhea and chlamydia testing provision. Apart from clinicians, community-based efforts may reduce stigma-based barriers to testing.

\section{Full Text}

Due to technical limitations, full-text HTML conversion of this manuscript could not be completed. However, the manuscript can be downloaded and accessed as a PDF.

\section{Tables}

Due to technical limitations, all tables are only available for download from the Supplementary Files section.

Figures 

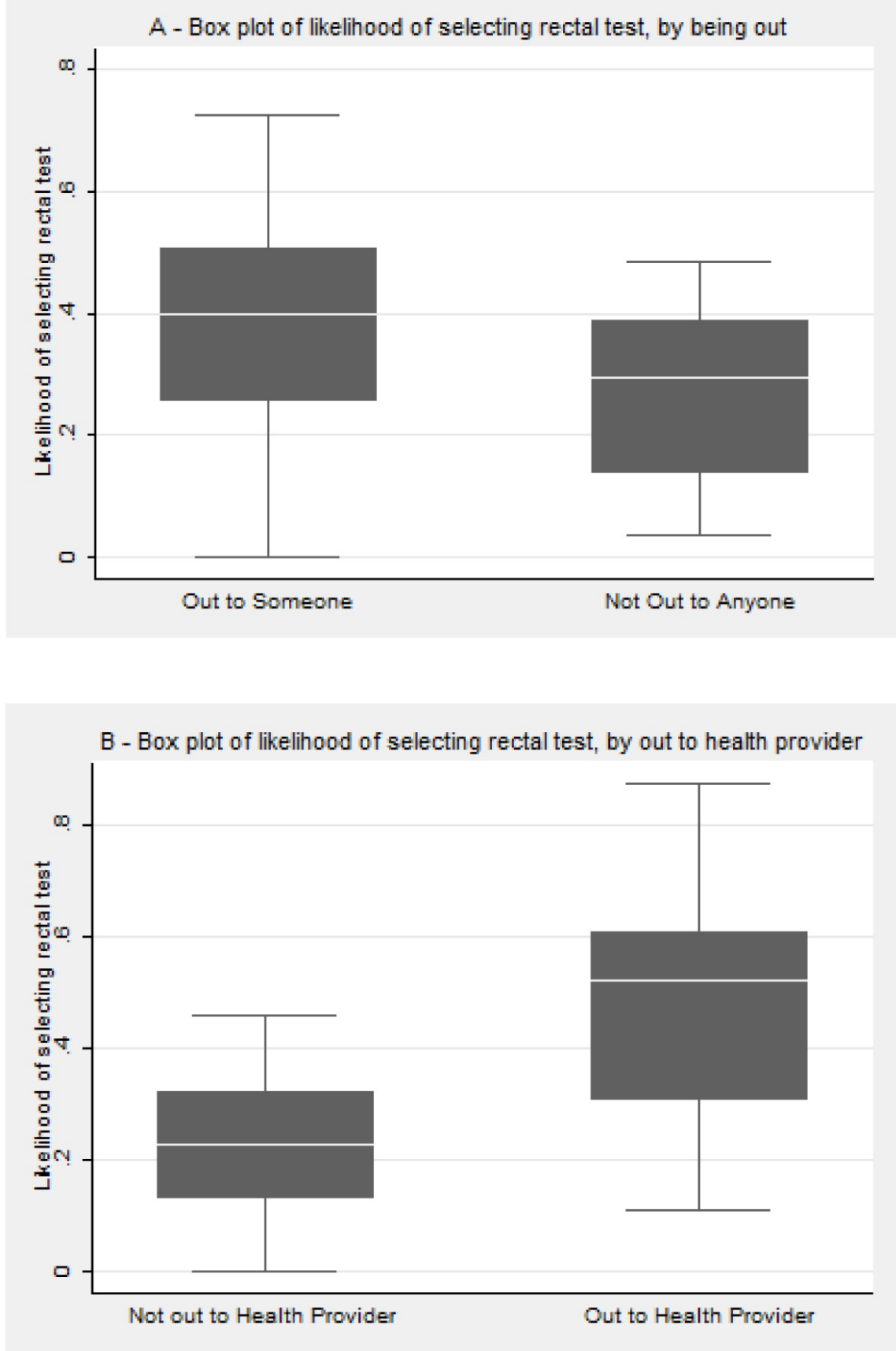

Figure 1

a) Box plot of likelihood of selecting rectal test, by being out; b) Box plot of likelihood of 
selecting rectal test, by out to health provider

\section{Supplementary Files}

This is a list of supplementary files associated with this preprint. Click to download.

Tables.pdf

Supp Survey.pdf

Table 1S.pdf 